\title{
Microscopic model of nano-scale particles removal in high pressure $\mathrm{CO}_{2}$-based solvents
}

\author{
Jiajue Chai ${ }^{\mathrm{a}}$, Xiaogang Zhang ${ }^{\mathrm{a}, *}$, Jin Huang ${ }^{\mathrm{a}}$, Xin Tan ${ }^{\mathrm{a}}$, Guoliang Dai ${ }^{\mathrm{b}, *}$ \\ a Department of Chemistry, Renmin University of China, Beijing, 100872, China \\ b National Microgravity Laboratory, Institute of Mechanics, Chinese Academy of Sciences, Beijing, 100080, China
}

\section{A R T I C L E I N F O}

\section{Article history:}

Received 11 June 2008

Received in revised form

22 December 2008

Accepted 24 December 2008

\section{Keywords:}

Equilibrium separation distance

Net adhesion force

High pressure

Supercritical $\mathrm{CO}_{2}$

Part cleaning

\begin{abstract}
A B S T R A C T
A physical model is presented to describe the kinds of static forces responsible for adhesion of nano-scale copper metal particles to silicon surface with a fluid layer. To demonstrate the extent of particle cleaning, equilibrium separation distance (ESD) and net adhesion force (NAF) of a regulated metal particle with different radii (10-300 $\mathrm{nm}$ ) on the silicon surface in $\mathrm{CO}_{2}$-based cleaning systems under different pressures were simulated. Generally, increasing the pressure of the cleaning system decreased the net adhesion force between spherical copper particle and silicon surface entrapped with medium. For $\mathrm{CO}_{2}+$ isopropanol cleaning system, the equilibrium separation distance exhibited a maximum at temperature $313.15 \mathrm{~K}$ in the regions of pressure space (1.84-8.02 MPa). When the dimension of copper particle was given, for example, $50 \mathrm{~nm}$ radius particles, the net adhesion force decreased and equilibrium separation distance increased with increased pressure in the $\mathrm{CO}_{2}+\mathrm{H}_{2} \mathrm{O}$ cleaning system at temperature $348.15 \mathrm{~K}$ under $2.50-12.67 \mathrm{MPa}$ pressure range. However, the net adhesion force and equilibrium separation distance both decreased with an increase in surfactant concentration at given pressure (27.6 or $27.5 \mathrm{MPa}$ ) and temperature (318 or $298 \mathrm{~K}$ ) for $\mathrm{CO}_{2}+\mathrm{H}_{2} \mathrm{O}$ with surfactant PFPE $\mathrm{COO}^{-} \mathrm{NH}_{4}{ }^{+}$or $\mathrm{DiF}_{8}-\mathrm{PO}_{4}{ }^{-} \mathrm{Na}^{+}$.
\end{abstract}

(C) 2008 Elsevier B.V. All rights reserved.

\section{Introduction}

Cleaning is a frequent operation unit to the semiconductor industry, ranging from improving raw material specifications to the high standards applied in chip manufacture. As the features in semiconductor devices shrink to nano-scale dimension, the ability of cleaning solutions to penetrate small features and contact the residues will become significantly more challenging. During solvent evaporation, capillary forces in such structures may collapse the sidewall and cause device failure [1,2]. Therefore, new concepts in semiconductor manufacture are required to challenge this complexity. Dense $\mathrm{CO}_{2}$, liquid and supercritical, has many desirable properties for cleaning semiconductors. It is inexpensive, essentially nontoxic, nonflammable and inert. Compared with liquid solvents, supercritical fluids are less viscous as a consequence of the great free volume, further more, solutes often diffuse $\sim 100$ times more rapidly. The surface tension of liquid carbon dioxide is $1.5 \mathrm{mN} / \mathrm{m}$ at $25^{\circ} \mathrm{C}$ and reaches zero at the critical point [3], where liquid droplets are no longer formed. These beneficial transport and interfacial properties accelerate the rates of mass transfer and promote the penetration of small features [4], and provide a sig-

\footnotetext{
* Corresponding authors. Tel.: +86-10-62516958.

E-mail address: zhang_xg@chem.ruc.edu.cn (X. Zhang).
}

nificant advantage over liquid and aqueous-based cleaning agents particularly with respect to cleaning high aspect ratio features $[5,6]$.

The numerous designs of dense $\mathrm{CO}_{2}$ cleaning and drying systems in semiconductor processing have been described previously [7-12]. Few of these studies focused on the microscopic behavior and physics models of particle removing in dense gas solvent. To understand the engineering and science of these new technological regimes, a physical model is required to gain a more in-depth knowledge of the study. Removal of particles and contaminations on surface with dense $\mathrm{CO}_{2}$ is governed by a complex array of factors that include van der Waals and electrostatic interactions, drag and lift forces, and phase changes that occur when the $\mathrm{CO}_{2}$ molecule impacts on the surface. Many authors have tried every effort to investigate different factors that affect the cleaning efficiency $[13,14]$. Garate [13] extended the DDM-model, which is developed for textile detergency based on aqueous solution, to dense $\mathrm{CO}_{2}$ system, and analyzed the variety factors including solvent flows, drag forces, slip flows, local micelle concentration gradients, mass transfer etc. on the cleaning and gave a satisfactory explanations for the real cleaning system based on $\mathrm{CO}_{2}$.

As many researchers pointed out, high solvent velocities impart high drag and shear forces that, if great enough, will overcome the particle adhesion and carry the particle into the bulk of detergent solutions [15-17]. However, the particles or residues in micronscale vias and trenches on the wafer have to be lifted into the bulk 


\begin{tabular}{|c|c|}
\hline \multicolumn{2}{|c|}{ Nomenclature } \\
\hline$A_{i i}$ & Hamaker constant of component $i$ \\
\hline$A_{132}$ & $\begin{array}{l}\text { Hamaker constant of a copper particle }(1) \text { and silica } \\
\text { surface (2) across a medium }\left(\mathrm{CO}_{2} / \text { polar solvent }\right)(3)\end{array}$ \\
\hline$d$ & $\begin{array}{l}\text { the sum of the particle radius and the particle- } \\
\text { surface separation distance }\left(z+r_{\mathrm{p}}\right)\end{array}$ \\
\hline$e$ & $\begin{array}{l}\text { dielectric constant of the fluid between the particle } \\
\text { and surface }\end{array}$ \\
\hline E & permittivity of free space \\
\hline$F_{\mathrm{vdw}}$ & van der Waals force \\
\hline$F_{\text {ca }}$ & classic Coulombic attraction \\
\hline$F_{\mathrm{cp}}$ & contact potential force \\
\hline$F_{\mathrm{g}}$ & gravitational force \\
\hline$F_{\mathrm{fb}}$ & fluid bridge force \\
\hline$F_{\text {cpe }}$ & capillary pressure effect force \\
\hline$g$ & acceleration due to gravity $\left(9.8 \mathrm{~m} / \mathrm{s}^{2}\right)$ \\
\hline$h$ & Planck's constant \\
\hline$k_{\mathrm{b}}$ & Boltzmann's constant \\
\hline$l$ & distance between charge centers \\
\hline$n_{i}$ & refractive index of component $i$ \\
\hline$n_{\text {vacuum }}$ & refractive index of vacuum \\
\hline$P$ & system pressure \\
\hline$P_{C}$ & capillary pressure \\
\hline$r_{\mathrm{P}}$ & particle radius \\
\hline$r_{\mathrm{M}}$ & meniscus radius \\
\hline$r_{\mathrm{K}}$ & Kelvin radius \\
\hline$R$ & gas constant $(8.314 \mathrm{~J} / \mathrm{mol} \mathrm{K})$ \\
\hline$T$ & absolute temperature \\
\hline$U_{\mathrm{pd}}$ & potential difference of particle and surface \\
\hline$V_{\mathrm{M}}$ & molar volume \\
\hline$z$ & particle-surface separation distance \\
\hline \multicolumn{2}{|c|}{ Greek letters } \\
\hline$\varepsilon_{i}$ & dielectric constant of component $i$ \\
\hline$\varepsilon_{\text {vacuum }}$ & dielectric constant of vacuum \\
\hline$\phi_{i}$ & volume fraction of component $i$ \\
\hline$\lambda$ & $\begin{array}{l}\text { characteristic wavelength of the van der Waals inter- } \\
\text { action }\end{array}$ \\
\hline$\theta$ & interfacial contact angle of meniscus \\
\hline$\sigma$ & $\begin{array}{l}\text { surface tension of entrapped fluid/solvent interface } \\
(\mathrm{N} / \mathrm{m})\end{array}$ \\
\hline$\rho_{\mathrm{c}}$ & critical density of $\mathrm{CO}_{2}$ \\
\hline$\rho$ & $\mathrm{CO}_{2}$ density under certain temperature and pressure \\
\hline$\rho_{\mathrm{g}}$ & density of dense gas (solvent) $\left(\mathrm{kg} / \mathrm{m}^{3}\right)$ \\
\hline$\rho_{\mathrm{p}}$ & density of particle $\left(\mathrm{kg} / \mathrm{m}^{3}\right)$ \\
\hline$v_{\mathrm{e}}$ & $\begin{array}{l}\text { maximum electronic ultraviolet adsorption fre- } \\
\text { quency }\end{array}$ \\
\hline
\end{tabular}

solvent before this process. Within these vias and trenches, solvent velocity approaches zero, so that the inside pores can be deemed approximately as a static-force system, in which imposed pressure helps lift particles from the substrate of pores due to the capillary effect. Mattews and Becnel's [18] calculations based on the idealized system ( $1 \mu \mathrm{m}$ graphite particle on a metal surface that is contaminated with a thin layer of $n$-dodecane) demonstrated that the use of dense $\mathrm{CO}_{2}$ in cleaning systems could significantly alter the static force balance on adhered particles. As the distance of particles from the pores-substrate increases, the influence of the bulk solvent increases, which in turn leads to a hydrodynamic removal process.

The solvation properties of $\mathrm{CO}_{2}$ are unique. It has no dipole moment; the outer oxygens are collinear with the central carbon. As a result, $\mathrm{CO}_{2}$ has far weaker van der Waals forces than those of hydrocarbon solvents even when it is compressed to $30 \mathrm{MPa}$. Consequently, many organic and inorganic substances are poorly soluble in $\mathrm{CO}_{2}$, which influences the applications. For cleaning systems, addition of an excess amount of solvent should lead to a wetting on the surface and particles to be easily removed. In some cases, small amounts of surfactant are needed to further augment the cleaning efficiency. For the removal of metal particles, small amount of chelating agent is useful for solvating metal or metal ion residues $[19,20]$. Theoretically, the introduction of excess amount of solvent into $\mathrm{CO}_{2}$-system forms a fluid bridge between microscopic particles and surfaces, which makes the static-force system more susceptible to pressure changes. This second fluid phase forms a well-defined phase interface with the bulk $\mathrm{CO}_{2}$ solvent. Because of the curvature of this phase interface, there is a pressure change within the entrapped fluid bridge, which can be calculated by the Kelvin equation [21]. The entrapped fluid brings two opposing forces on the particle, one adding to the adsorption forces, the other acting to counteract those forces [22].

The objective of this work is to investigate the effect of pressure on static forces for removal of nano-scale metal particles by simulation in hierarchy of the $\mathrm{CO}_{2}$-based solvents systems and develop an understanding of the mechanism of the metal residue removal. Equilibrium separation distance (ESD) and net adhesion force (NAF) of a regulated metal particle with different radii from the silicon surface under different pressures, temperatures can be determined. The metal residual selected was copper.

\section{Model description and procedure}

In the general case, adsorption forces of particles adhering to substrate consist of van der Waals force, classical Coulombic attraction (electrostatic image force), contact potential force, and fluid bridge force. Gravitational force also adds to the adsorption force. These forces all together act as an obstacle for removing particles adhering to the surface. With regard to the fluid bridge entrapped between the particle and the surface, there is an opposing force or the capillary pressure due to the curvature of the interface acting against the adsorption force. In the model, the particle is taken to be a perfect sphere close to a perfectly flat smooth surface. Both the particle and the surface have homogeneous physical properties. No deformation of the particle and substrate are considered (Fig. 1).

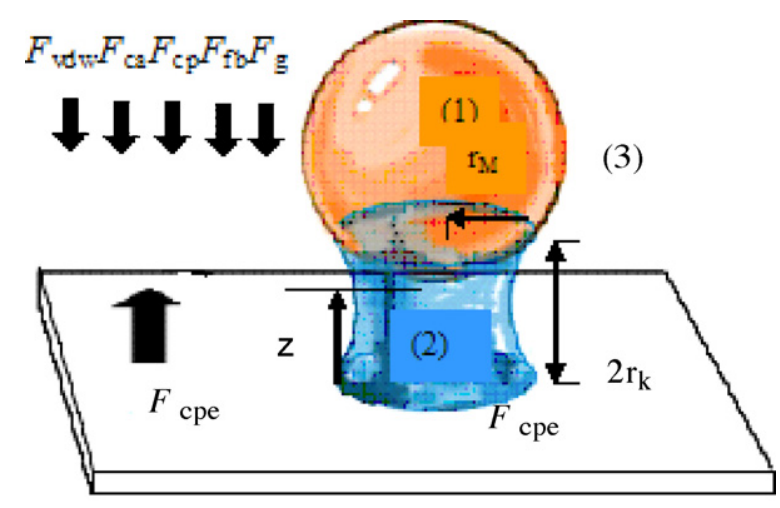

(4)

Fig. 1. Graphical description of metal particle and static equilibrium in solvent assisted $\mathrm{CO}_{2}$-systems. (1) Particle (Cu), (2) entrapped fluid, (3) bulk solvent, and (4) substrate. $r_{\mathrm{M}}$ is the meniscus radius, $r_{\mathrm{K}}$ is the Kelvin radius, $z$ is the particle-surface separation distance. The five downward arrows represent van der Waals force $F_{\mathrm{vdw}}$, classical Coulombic attraction force $F_{\mathrm{ca}}$, contact potential force $F_{\mathrm{cp}}$, fluid bridge force $F_{\mathrm{fb}}$, and gravitational force $F_{\mathrm{g}}$. The upward arrow represents capillary pressure effect force $F_{\text {cpe }}$. 


\section{1. van der Waals force}

The van der Waals interactions between bodies of material, arising from the interaction of oscillating dipoles in the interatomic bonds of each body, manifest themselves in various aspects of behavior ranging from the determination of surface energies, and consequently wetting behavior, to the stability of colloidal suspensions and emulsions [23]. The Hamaker constant is convenient for representing these interactions. The Hamaker constant of pure substance $i$ depends on its dielectric constant $(\varepsilon)$ and refractive index $(n)$ of the particles and the nature of the solvating medium. The Hamaker constant $A_{i i}$ can be estimated using a simplification of the Lifshift theory [24]

$A_{i i}=\frac{3}{4} k_{\mathrm{b}} T\left(\frac{\varepsilon_{i}-\varepsilon_{\text {vacuum }}}{\varepsilon_{i}+\varepsilon_{\text {vacuum }}}\right)^{2}+\frac{3 h v_{\mathrm{e}}}{16 \sqrt{2}} \frac{\left(n_{i}^{2}-n_{\text {vacuum }}^{2}\right)^{2}}{\left(n_{i}^{2}+n_{\text {vacuum }}^{2}\right)^{3 / 2}}$

in this case, $\varepsilon$ is the dielectric constant, $n$ is the refractive index, $k_{\mathrm{b}}$ is Boltzmann's constant, $T$ is absolute temperature, $h$ is Planck's Constant, $v_{\mathrm{e}}$, is the maximum electronic ultraviolet adsorption frequency typically taken to be $3 \times 10^{15} \mathrm{~s}^{-1}$ [24]. The density dependence of the refractive index and dielectric constant must be accounted for when the supercritical fluids are used as the solvent. Using Eq. (1), $\mathrm{CO}_{2}$ interactions across a vacuum can be determined by setting $\varepsilon_{\text {vacuum }}$ and $n_{\text {vacuum }}$ to 1 . The equilibrium adsorption of nano-particles in a pore onto the silicon surface may be expected to be driven primarily by the chemical potential of the nano-particles in the solvent and long-ranged van der Waals forces between the particles and silicon surface. For simplicity, we treat the silicon surface as a flat wall. The Hamaker constant $A_{132}$, for a copper particle (1) and silicon surface (2) across a medium ( $\mathrm{CO}_{2} /$ solvent) (3) may be approximated by [23]:

$A_{132}=\left(\sqrt{A_{11}}-\sqrt{A_{33}}\right)\left(\sqrt{A_{22}}-\sqrt{A_{33}}\right)$

The $A_{33}$ was determined for the binary solvent mixture $\left(\mathrm{CO}_{2} /\right.$ solvent $)$ from Eq. (1) along with the Lorenz-Lorenz mixing rule:

$\frac{\left(n_{\text {mix }}-1\right)^{2}}{\left(n_{\text {mix }}+2\right)^{2}}=\sum \frac{\phi_{i}\left(n_{i}-1\right)^{2}}{\left(n_{i}+2\right)^{2}}$

where $\phi_{i}$ is the volume fraction of component $i$. An equivalent expression for the dielectric constant is obtained by replacing $n$ with $\sqrt{\varepsilon}$ in Eq. (3). The $n$ and $\varepsilon$ for $\mathrm{CO}_{2}$ were obtained as a function of density from the following $[25,26]$ :

$\frac{n^{2}-1}{n^{2}+2}=0.07016 \rho_{r}+1.412 \times 10^{-4} \rho_{\mathrm{r}}^{2}-3.171 \times 10^{-4} \rho_{\mathrm{r}}^{3}$

$\varepsilon-1=0.2386 \rho_{\mathrm{r}}+0.02602 \rho_{\mathrm{r}}^{2}$

where $\rho_{\mathrm{r}}=\rho / \rho_{\mathrm{c}}, \rho_{\mathrm{c}}$ is the critical density of $\mathrm{CO}_{2}$, usually taken to be $333.5 \mathrm{~kg} / \mathrm{m}^{3}$.

Because of the presence of a medium between the particle and substrates, the magnitude of the van der Waals force is decreased or retarded. The modified equation for van der Waals force between a spherical particle and surface entrapped with medium was derived by Chen and Anandarajah [27]:

$$
\begin{aligned}
F_{\mathrm{vdw}}= & A_{132}\left[F_{1}(d)+F_{2}(d)\right] \\
F_{1}(d)= & \frac{2}{3 r_{\mathrm{P}}\left[\left(d / r_{\mathrm{P}}\right)^{2}-1\right]} \\
F_{2}(d)= & -\frac{2\left(d+c-2 r_{\mathrm{P}}\right)}{3 c^{4}}\left(d+c+r_{\mathrm{P}}\right)^{2} \ln \left(d+c+r_{\mathrm{P}}\right) \\
& +\frac{2\left(d+c+2 r_{\mathrm{P}}\right)}{3 c^{4}}\left(d+c-r_{\mathrm{P}}\right)^{2} \ln \left(d+c-r_{\mathrm{P}}\right)
\end{aligned}
$$

$$
\begin{aligned}
& -\left[\frac{2\left(d+2 r_{\mathrm{P}}\right)}{3 c^{4}}\left(d-r_{\mathrm{P}}\right)^{2}+\frac{2\left(d+r_{\mathrm{P}}\right)}{c^{3}}\left(d-r_{\mathrm{P}}\right)+\frac{2 d}{c^{2}}+\frac{2}{3 c}\right] \\
& \times \ln \left(d-r_{\mathrm{P}}\right)+\left[\frac{2\left(d-2 r_{\mathrm{P}}\right)}{3 c^{4}}\left(d+r_{\mathrm{P}}\right)^{2}+\frac{2\left(d-r_{\mathrm{P}}\right)}{c^{3}}\left(d+r_{\mathrm{P}}\right)\right. \\
& \left.+\frac{2 d}{c^{2}}+\frac{2}{3 c}\right] \ln \left(d+r_{\mathrm{P}}\right)-\frac{4 d r_{\mathrm{P}}}{3 c^{3}}-\frac{8 r_{\mathrm{P}}}{3 c^{2}}-\frac{4 d r_{\mathrm{P}}}{3 c\left(d^{2}-r_{\mathrm{P}}^{2}\right)}
\end{aligned}
$$

In this case, $c=3.1 \lambda / 2 \pi, \lambda$ is the characteristic wavelength of the van der Waals interaction which is taken to be $100 \mathrm{~nm}$ [27]. With $\lambda=100 \mathrm{~nm}, \mathrm{c}$ is computed to be $49.363 \mathrm{~nm}$. In Eqs. (6-8), $d$ is the sum of the particle radius $r_{\mathrm{p}}$ and the particle-surface separation distance $z$, where $z$ is the distance between the underside edge of the particle and the bottom of the substrate. $F_{1}(d)$ represents nonretarded contributions; $F_{2}(d)$ represents the retardation effects.

\subsection{Electrostatic attraction force}

Two types of electrostatic interactions that cause particles to adhere to surfaces exist in this model. The first type is owing to bulk excessive charge in the particle or surface, which accounts for the classical Coulombic attraction between the particle and the surface, also named electrostatic image force, represented by [28]:

$F_{\mathrm{ca}}=\frac{4 \pi E r_{\mathrm{P}}^{2} U_{\mathrm{BC}}^{2}}{e l^{2}}$

In this case, $e$ is the dielectric constant of the fluid between the particle and surface, $E$ is the permittivity of free space, and $l$ is the distance between charge centers. For the case of a particle and a surface, $l$ is taken to be twice the particle radius plus the separation distance $\left(2\left(r_{\mathrm{P}}+z\right)\right)$ [29]. $U_{\mathrm{BC}}$ is the bulk charge in volts.

The second type of form of electrostatic interaction is the result of differences in local energy states and electron work functions, resulting in a contact potential difference which establishes a double layer charge region and a resulting adhesion force, given by [28]:

$F_{\mathrm{cp}}=\frac{\pi E r_{\mathrm{P}} U_{\mathrm{PD}}^{2}}{z}$

where $r_{\mathrm{P}}$ is the particle radius, $z$ is the separation distance, and $U_{\mathrm{PD}}$ is potential difference in volts.

\subsection{Gravitational force}

The gravitational force can be given by:

$F_{\mathrm{g}}=\frac{4}{3} \pi r_{\mathrm{P}}^{3} g\left(\rho_{\mathrm{P}}-\rho_{\mathrm{g}}\right)$

where $\rho_{\mathrm{P}}$ denotes the density of copper, $\rho_{\mathrm{g}}$ denotes the vapor phase density of the solvent, and $g$ is the acceleration of gravity.

\subsection{Fluid bridge force}

The interface of entrapped fluid bridge with the bulk solvent creates two opposing forces, one of which is the fluid bridge force, called surface tension force as well, acting to add to the total adhesion force. This force is given by [28]:

$F_{\mathrm{fb}}=4 \sigma \pi r_{\mathrm{M}}$

In this case, $\sigma$ is the surface tension and $r_{M}$ is the meniscus radius. The meniscus radius is defined as the perpendicular length from the particle centerline to where the phase interface contacts the particle. The particle centerline is the line passing through the particle center and perpendicular to the surface (Fig. 1). 


\subsection{Capillary pressure effect force}

The other aspect of the interface effect mentioned above is the capillary pressure effect force, which acts to lift the particle from the surface. This effect has been related to capillary effects in narrow pores.

The Kelvin equation has been used extensively to describe the pressure increase within a particle-surface entrapped fluid. This pressure increase is analogous to the capillary pressure rise and can be calculated by the Kelvin equation [21]:

$\ln \left(\frac{P_{\mathrm{C}}}{P}\right)=\frac{2 \sigma V_{\mathrm{M}} \cos \theta}{R T r_{\mathrm{K}}}$

In this case, $\sigma$ is the surface tension; $r_{\mathrm{K}}$, the Kelvin radius, is approximately half of the perpendicular distance from the surface to the point of contact between the fluid interface and the particle; $V_{\mathrm{M}}$ is the molar volume; $P$ is the system pressure; $P_{\mathrm{C}}$ is the capillary pressure (i.e., pressure within the entrapped fluid); $\theta$ is the interfacial contact angle; $R$ is the gas constant; and $T$ is the system temperature. Molar volume concerns phase compositions and liquid phase density, and is estimated by:

$V_{\mathrm{M}}=\mathrm{MW} / \rho_{\mathrm{L}}$

where $\rho_{\mathrm{L}}$ is the liquid phase density of the $\mathrm{CO}_{2}$ /solvent mixture under a certain pressure and temperature. $\mathrm{MW}$ is the molecular weight of liquid phase of the $\mathrm{CO}_{2}$ /solvent mixture, calculated by:

$\mathrm{MW}=\mathrm{MW}\left(\mathrm{CO}_{2}\right) \times \mu_{\mathrm{L}}\left(\mathrm{CO}_{2}\right)+\mathrm{MW}($ solvent $) \times \mu_{\mathrm{L}}$ (solvent $)$

where $\mu_{\mathrm{L}}\left(\mathrm{CO}_{2}\right)$ and $\mu_{\mathrm{L}}$ (solvent) are the molar fraction of $\mathrm{CO}_{2}$ and that of solvent in the $\mathrm{CO}_{2}$ /solvent mixtures of liquid phase.

For an interface with a contact angle of less than $90^{\circ}$, the pressure within the entrapped fluid is greater than that of the ambient pressure. In these cases, the particle experiences a force away from the entrapped fluid which can be calculated using mensuration formulas:

$F_{\text {cpe }}=\left(P-P_{\mathrm{C}}\right)\left[2 \pi r_{\mathrm{P}}\left(r_{\mathrm{P}}-\sqrt{r_{\mathrm{P}}^{2}-r_{\mathrm{M}}^{2}}\right)\right]$

Each group of calculation followed the same route to reach a result. The first step was to solve Eqs. (1-5) to work out the parameters, such as Hamaker constant $A_{132}$, dielectric constant of fluid mixture $\varepsilon$, and refractive index of the fluid mixture $n$. This optimizing process utilized the trial and error method, so as to reach an appropriate ESD, where the capillary pressure effect force offsets the total adsorption force completely. Eqs. (6-16) were used to calculate the static forces of a particle on substrate under different pressures and temperatures. The solution of them employed Mathematica to obtain a series of data. Static forces at its ESD were calculated. When the particle reaches its equilibrium distance, the downward forces imposed on it equal to the upward forces indicating that a particle will be stable to minor perturbations in separation distance [18]. The force balance on the particle was calculated without any dynamic removal forces such as solvent drag, slipping or rolling.

Bulk charge and potential difference vary much according to particle type, substrates as well as cleaning media; these values can be taken to optimize the magnitude of the Coulombic forces in the model [28]. We selected the $\mathrm{CO}_{2}$-isopropanol as the model system, and chose a series of bulk charge and potential difference, and found if there existed a stable ESD. The calculation results demonstrated that there existed a stable ESD $\left(10^{-10} \mathrm{~m}\right)$ only setting bulk charge and potential difference as 6 and $0.1 \mathrm{~V}$, respectively. For other values, the ESD was either 0 or of too small magnitude $\left(10^{-11}\right.$ or $\left.10^{-12} \mathrm{~N}\right)$, making separation impossible.

In this study, we assume that the residues are all complete sphere particles with identical radius of $10-300 \mathrm{~nm}$. The entrapped
Table 1

Model parameters for Fig. 2(a and b).

\begin{tabular}{lc}
\hline Parameter & Value \\
\hline Particle bulk charge, $U_{\mathrm{BC}}(\mathrm{V})$ & 6.0 \\
Material potential difference, $U_{\mathrm{PD}}(\mathrm{V})$ & 0.1 \\
Vacuum permittivity, $E(\mathrm{~F} / \mathrm{m})$ & $8.854 \times 10^{-12}$ \\
Interfacial contact angle $\left(^{\circ}\right)$ & 0 \\
Critical density of $\mathrm{CO}_{2}\left(\mathrm{~kg} / \mathrm{m}^{3}\right)$ & 333.5 \\
Density of copper $\left(\mathrm{kg} / \mathrm{m}^{3}\right)$ & 8930 \\
Dielectric constant of isopropanol at $313.15 \mathrm{~K}$ & 16.2 \\
Refractive index of isopropanol at $313.15 \mathrm{~K}$ & 1.37 \\
\hline
\end{tabular}

fluid is idealized to entirely wet both the particle and the surface $\left(0^{\circ}\right.$ contact angle). Due to the complexity and method limitation of predicting or observing the meniscus radius, it is often taken as the free variable by setting a fixed scale between particle radius $\left(r_{\mathrm{P}}\right)$ and meniscus radius $\left(r_{\mathrm{M}}\right)$. In this case, a dimensionless radius $r_{\mathrm{M}} / r_{\mathrm{P}}$ was taken to be the value of 0.1 . Using the similar method of setting bulk charge and potential difference mentioned above, we calculated ESD under different $r_{\mathrm{M}} / r_{\mathrm{P}}$ ratios $0.1,0.15,0.2,0.25,0.3$, and 0.4 for the particles with different radius, finding that only the value of 0.1 exhibited a stable ESD.

\section{Results and discussion}

\section{1. $\mathrm{CO}_{2}+$ isopropanol system}

Isopropanol is well accepted as the most effective cosolvent for semiconductor cleaning [30]. The calculation was done for particles with different radius in $\mathrm{CO}_{2}$-isopropanol medium at $313.15 \mathrm{~K}$ and pressures from 1.84 to $8.14 \mathrm{MPa}$. In the calculation, the data of isopropanol and $\mathrm{CO}_{2}$ were taken from references [3,31,32]. Surface tension, vapor liquid equilibrium phase compositions, phase densities were taken or calculated according to the references [33-35]. Detailed model parameters for Fig. 2(a and b) are listed in Table 1. As there was no accurate data concerning the interfacial tension of $\mathrm{CO}_{2}$-isopropanol, in this study, the interfacial tension of $\mathrm{CO}_{2}$-isopropanol under different pressures was substituted approximately by employing the $\mathrm{CO}_{2}$-ethanol system, which depends on the carbon dioxide density and was almost independent of temperature, and could be calculated by following equation [36]

$\sigma=1.759 \times 10^{-4} \times \rho_{\mathrm{CO}_{2}}^{2}-1.127 \times 10^{-1} \times \rho_{\mathrm{CO}_{2}}+1.898 \times 10^{1}$

In each set of calculations, the NAF and ESD were computed. Fig. 2( $a$ and $b$ ) gave the NAF and ESD as a function of the pressure and particle radius, respectively, and related data listed in Table S1( $a$ and $b)$. The NAF is the sum of all of the forces which adhere the particle to the substrate. At given pressure, NAF and ESD increased with the increase of particle radius. In current model, the meniscus radius was taken as the free variable, and the dimensionless radius value $\left(r_{M} / r_{P}\right)$ was fixed to 0.1 .

For a given particle radius, the NAF decreased with increased pressure. A significant decrease of the NAF could result from the decrease of fluid bridge force $\left(F_{\mathrm{fb}}\right)$ which decreased with increased pressure (shown in Table S2). Unlike the trends of NAF, the value of ESD had a maximum in the pressure ranges, and the maximum shifted to low pressure range with decreased particle radius. The value of ESD trended to relatively small value $(<4 \AA)$ as the pressure was larger than 7.0 MPa when the particle radius smaller than $50 \mathrm{~nm}$, suggesting that the system could not achieve the stable equilibrium separating state, and that it was impossible for these particles to be removed under these pressure conditions [18].

ESD means the distance at which the downward forces imposed on the particles equal to the upward forces. From Eqs. (13-17), we can see that surface tension and molar volume of the entrapped fluid decreased with increased pressure. The reduction in surface 

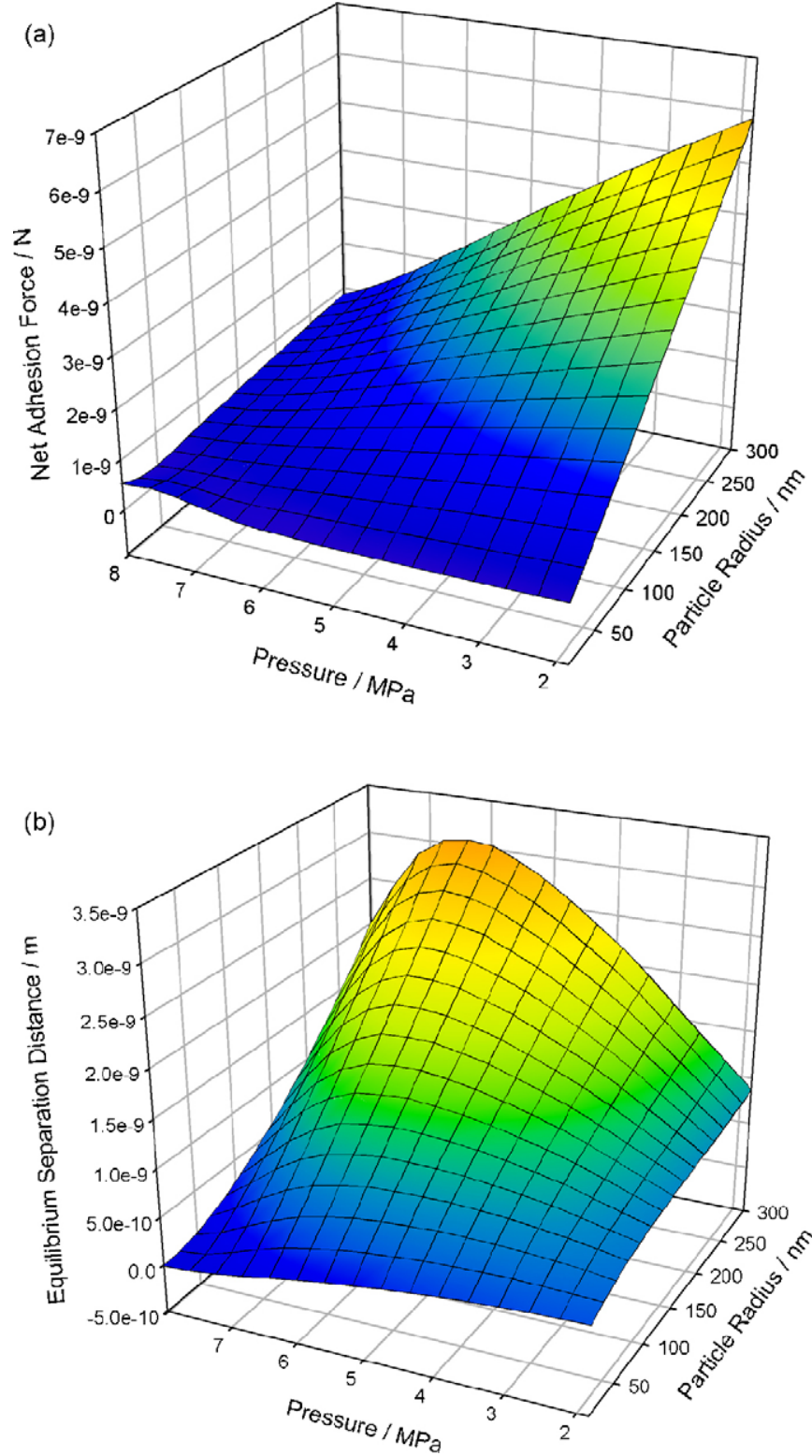

Fig. 2. (a) Net adhesion force (NAF) between spherical Cu particle and silicon surface entrapped with isopropanol medium as a function of the pressure and particle radius in $\mathrm{CO}_{2}$ + isopropanol at $313.15 \mathrm{~K}$. (b) Equilibrium separation distance (ESD) between spherical $\mathrm{Cu}$ particle and silicon surface entrapped with isopropanol medium as a function of the pressure and particle radius in $\mathrm{CO}_{2}+$ isopropanol at $313.15 \mathrm{~K}$.

Table 2

Parameters for Fig. 3.

\begin{tabular}{lc}
\hline Parameter & Value \\
\hline Particle bulk charge, $U_{\mathrm{BC}}(\mathrm{V})$ & 6.0 \\
Material potential difference, $U_{\mathrm{PD}}(\mathrm{V})$ & 0.1 \\
Vacuum dielectric constant, $E(\mathrm{~F} / \mathrm{m})$ & $8.854 \times 10^{-12}$ \\
Interfacial contact angle $\left({ }^{\circ}\right)$ & 0 \\
Critical density of $\mathrm{CO}_{2}\left(\mathrm{~kg} / \mathrm{m}^{3}\right)$ & 833.5 \\
Density of copper $\left(\mathrm{kg} / \mathrm{m}^{3}\right)$ & 6930 \\
Dielectric constant of water at $348.15 \mathrm{~K}$ & 1.32 \\
Refractive index of water at $348.15 \mathrm{~K}$ &
\end{tabular}

tension decreases the fluid bridge force $F_{\mathrm{fb}}$, while the decrease in surface tension and the molar volume of the entrapped fluid increases the capillary pressure effect force $F_{\text {cpe. The data listed }}$ in Table S1 showed that the fluid bridge force $\left(F_{\mathrm{fb}}\right)$ was the main proportion of NAF in the lower pressure stage, the decrease of $F_{\mathrm{fb}}$

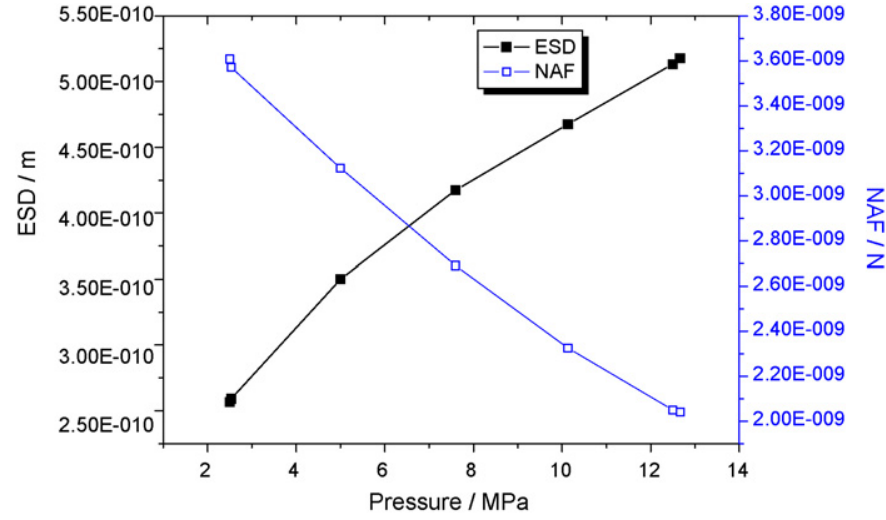

Fig. 3. Equilibrium separation distance (ESD) and net adhesion force (NAF) of $50 \mathrm{~nm}$ copper particle in $\mathrm{CO}_{2}-\mathrm{H}_{2} \mathrm{O}$ system, at $348.15 \mathrm{~K}$ and under pressures from 2.50 to $12.67 \mathrm{MPa}$.

lead to an increase of ESD with pressure increasing. In the higher pressure stage, the proportion of $F_{\mathrm{fb}}$ in NAF decrease, the quantitative difference of all downward forces (except for gravitational force $\left(F_{\mathrm{g}}\right)$ ) becomes insignificant. The value of ESD curved to the maximum at which the kinds of forces reach to the compromise.

\section{2. $\mathrm{CO}_{2}+\mathrm{H}_{2} \mathrm{O}$ system without and with surfactant}

Besides $\mathrm{CO}_{2}$-isopropanol system, $\mathrm{CO}_{2}-\mathrm{H}_{2} \mathrm{O}$ with and without surfactant systems were also selected. We took $50 \mathrm{~nm} \mathrm{Cu}$ particles as the sample to calculate the NAF and ESD under different pressures for the cleaning systems. Interfacial tensions of $\mathrm{CO}_{2}+\mathrm{H}_{2} \mathrm{O}$ and $\mathrm{CO}_{2}+\mathrm{H}_{2} \mathrm{O}+$ surfactant were taken from the references [37-40], respectively. The dielectric constant and refractive index of pure water were taken from the reference [41]. Relative parameters
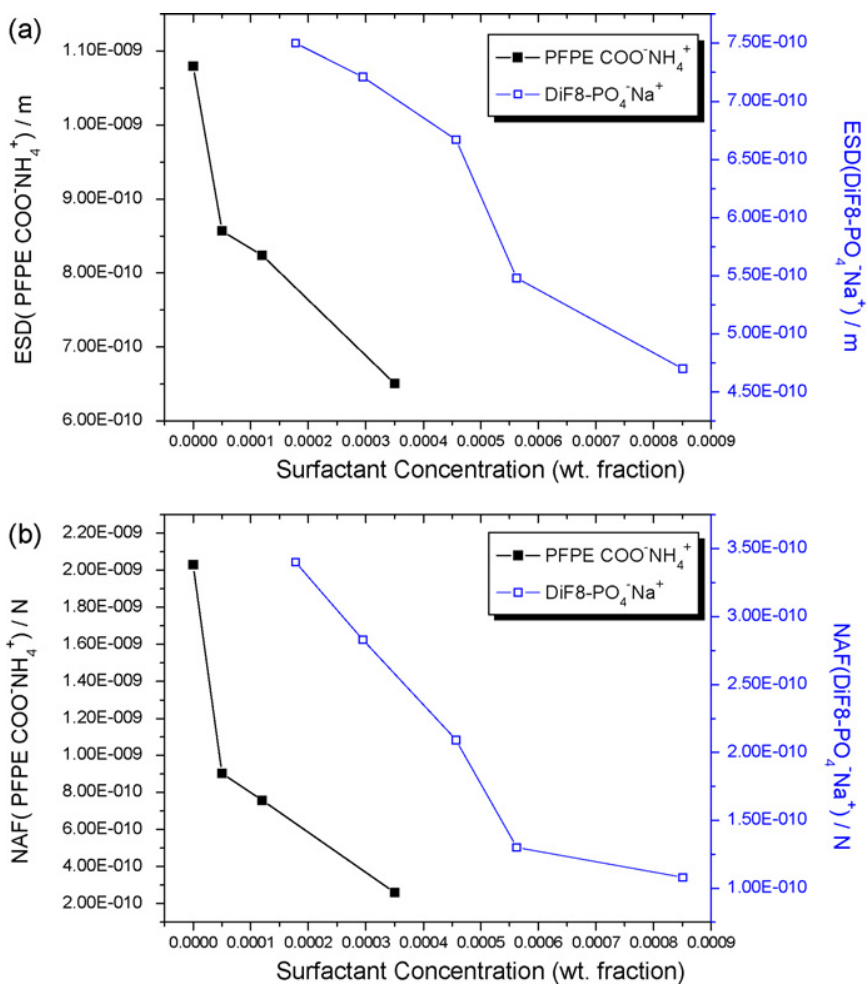

Fig. 4. ESD and NAF as a function of the ionic surfactant (PFPE $\mathrm{COO}^{-} \mathrm{NH}_{4}{ }^{+}$or $\mathrm{DiF}_{8}$ $\mathrm{PO}_{4}{ }^{-} \mathrm{Na}^{+}$) concentration for $50 \mathrm{~nm}$ copper particle in $\mathrm{CO}_{2}+\mathrm{H}_{2} \mathrm{O}$ system under $318.0 \mathrm{~K}$ and 27.6 MPa for PFPE COO- $\mathrm{NH}_{4}{ }^{+}$, and $298.0 \mathrm{~K}$ and $27.5 \mathrm{MPa}$ for $\mathrm{DiF}_{8}-\mathrm{PO}_{4}{ }^{-} \mathrm{Na}^{+}$. Other parameters are identical to the system $\mathrm{CO}_{2}+\mathrm{H}_{2} \mathrm{O}$. 
listed in Table 2. The computation was taken in the system of $\mathrm{CO}_{2}$ $\mathrm{H}_{2} \mathrm{O}$ vapor-liquid equilibrium at $348.15 \mathrm{~K}$ under different pressures from 2.50 to $12.67 \mathrm{MPa}$. Fig. 3 demonstrated that high pressure could increase the particle-substrate separation distance in the regions of pressures studied and this effect might be coupled with a decrease in the NAF for the $\mathrm{CO}_{2}-\mathrm{H}_{2} \mathrm{O}$ system without surfactant, suggesting the particles would be removed more easily at high pressure.

The use of surfactants in $\mathrm{H}_{2} \mathrm{O}-\mathrm{CO}_{2}$ system has the potential to facilitate particle disengagement from surfaces. In this case, the replacement of the high interfacial tension between the substrate and particle with two new interfaces with lower interfacial tensions is energetically favorable. The surfactant helps overcome the adhesive forces that bond the particle to the surface. From Fig. 4, we could see that addition of surfactant could significantly reduce the surface tension, the NAF of $\mathrm{H}_{2} \mathrm{O}-\mathrm{CO}_{2}$ system with surfactant (PFPE $\mathrm{COO}^{-} \mathrm{NH}_{4}{ }^{+}$or $\mathrm{DiF}_{8}-\mathrm{PO}_{4}{ }^{-} \mathrm{Na}^{+}$) was much less, and the value of NAF decreased with the increase of surfactant. The surfactants can also provide steric stabilization for particles suspended in $\mathrm{CO}_{2}$, as the hydrophobic tails extend outwards into $\mathrm{CO}_{2}$. These cooperative effects improve the efficient removal for the particles. Meanwhile, we could see that the ESD also decreased with increasing surfactant concentration, suggesting that adding surfactant into cleaning system facilitated the particles removal, but it was still a challenge to lift these particles out of the trench with large aspect ratio. In this work, we calculated the NAF and ESD in the case of $\mathrm{H}_{2} \mathrm{O}-\mathrm{CO}_{2}$ system with anionic surfactant (PFPE $\mathrm{COO}^{-} \mathrm{NH}_{4}{ }^{+}$or $\left.\mathrm{DiF}_{8}-\mathrm{PO}_{4}{ }^{-} \mathrm{Na}^{+}\right)$. Studies on more kinds of surfactants are now in progress.

\section{Conclusions}

A model has been presented to demonstrate pressure effect on the kinds of static forces of nano-scale particle adhesion on the solid surface with a fluid layers on the substrate. Using realistic parameters, the net adhesion force and equilibrium separation distance in $\mathrm{CO}_{2}+$ isopropanol, $\mathrm{CO}_{2}+\mathrm{H}_{2} \mathrm{O}$ with and without surfactant cleaning systems were calculated. The calculated results showed that high pressure could impose enough capillary pressure effect beneath a particle to compete with the net adhesion force and increase the particle surface separation distance. Although an increase in the pressure facilitates the particle removal, the equilibrium separation distance exhibits a maximum with respect to the pressure in $\mathrm{CO}_{2}+$ isopropanol cleaning system. Increasing pressure facilitate the particle removal in the $\mathrm{CO}_{2}+\mathrm{H}_{2} \mathrm{O}$ solution because the net adhesion force decrease and equilibrium separation distance increase with increased pressure in this cleaning system. Addition of surfactant (PFPE COO ${ }^{-} \mathrm{NH}_{4}{ }^{+}$or $\mathrm{DiF}_{8}-\mathrm{PO}_{4}{ }^{-} \mathrm{Na}^{+}$) into the $\mathrm{CO}_{2}+\mathrm{H}_{2} \mathrm{O}$ cleaning system can result in the net adhesion force decrease significantly, yet the equilibrium separation distance decrease also with an increase in surfactant concentration. This model is helpful to design and analysis of high pressure $\mathrm{CO}_{2}$-based precision cleaning systems.

\section{Acknowledgement}

The authors thank gratefully for financial support provided by the National Nature Science Foundation of China (20576140, 20603043)

\section{Appendix A. Supplementary data}

Supplementary data associated with this article can be found, in the online version, at doi:10.1016/j.supflu.2008.12.012.

\section{References}

[1] P.G. Clark, B.D. Schwab, J.W. Butternaugh, H.J. Martinez, P.J. Wolf, Cleaning and restoring k value of porous MSQ films, Semicond. Int. 26 (2003) 46-52.

[2] G.B. Jacobson, B. Palmer, R. Brown, D. Dreissig, D. Yellowaga, M. Biberger, Clean ing of photoresist and etch residue from dielectrics using supercritical $\mathrm{CO}_{2}$, in: Wafer Clean and Surface Prep Workshop 2003, International Sematech, Austin, 2003.

[3] J.A. Dean, Lange's Handbook of Chemistry, fifteenth ed., McGraw-Hill, New York, 1998.

[4] Y. Fukushima, H. Wakayama, Nanoscale casting using supercritical fluid, J. Phys. Chem. B 103 (1999) 3062-3064.

[5] Y. Jincao, M.A. Matthews, Prevention of photoresist pattern collapse by using liquid carbon dioxide, Ind. Eng. Chem. Res. 40 (2001) 5858-5860.

[6] C.F. Tsang, C.K. Chang, A study of post-etch wet clean on electrical and reliability performance of $\mathrm{Cu} /$ low $k$ interconnections, Microelectron. Reliab. 45 (2005) 517-525.

[7] D.L. Goldfarb, J.J. Pablo, P.F. Nealey, Aqueous-based photoresist drying using supercritical carbon dioxide to prevent pattern collapse, J. Vac. Sci. Technol. B 18 (2000) 3313-3317.

[8] H. Namatsua, Supercritical resist drying for isolated nanoline formation, J. Vac. Sci. Technol. B 19 (2001) 2709-2712.

[9] H. Namatsua, Supercritical drying for water-rinsed resist systems, J. Vac. Sci. Technol. B 18 (2000) 3308-3312.

[10] X.Zhang, J.Q. Pham, N. Ryza, P.F. Green, K.P.Johnston, Chemical-mechanical photoresist drying in supercritical carbon dioxide with hydrocarbon surfactants, $\mathrm{J}$ Vac. Sci. Technol. B 22 (2004) 818-825.

[11] X. Zhang, J.Q. Pham, Water-in-carbon dioxide microemulsions for removing post-etch residues from patterned porous low- $k$ dielectrics, J. Vac. Sci. Technol. B 21 (2003) 2590-2598.

[12] J.A. Keagy, X. Zhang, K.P. Johnston, Cleaning of patterned porous low-k dielectrics with water, carbon dioxide and ambidextrous surfactants, J. Supercrit. Fluids 39 (2006) 277-285.

[13] M.P. Garate, Ph.D. Dissertation, Delft University of Technology, Delft, 2004

[14] Y. Zhu, S. Granick, No-slip boundary condition switches to partial slip when fluid contains surfactant, Langmuir 18 (2002) 10058-10063.

[15] J.A. Kabin, A.E. Sáez, C.S. Grant, R.G. Carbonell, Removal of organic films from rotating disks using aqueous solutions of nonionic surfactants: film morphology and cleaning mechanisms, Ind. Eng. Chem. Res. 35 (1996) 44944506.

[16] S. Thomas, A. Faghri, W. Hankey, Experimental analysis and flow visualization of a thin liquid film on a stationary and rotating disk, J. Fluids Eng. 113 (1991) 73-80.

[17] J.A. Shaeiwitz, A.F. Chan, E.L. Cussler, D.F. Evans, The mechanism of solubilization in detergent solutions, J. Colloid Interface Sci. 84 (1981) 47-56.

[18] M.A. Matthews, J. Becnel, Effect of pressure on the static forces of micron-scale particle adhesion, Ind. Eng. Chem. Res. 39 (2000) 4481-4486.

[19] A.V. Yazdi, E.J. Beckman, Design, synthesis, and evaluation of novel, highly $\mathrm{CO}_{2}$ soluble chelating agents for removal of metals, Ind. Eng. Chem. Res. 35 (1996) 3644-3652.

[20] E. Bok, D. Kelch, K.S. Schumacher, Supercritical fluids for single wafer cleaning, Solid State Technol. 35 (1992) 117-120.

[21] C.N. Satterfield, Heterogeneous Catalysis in Practice, McGraw-Hill, New York, 1980.

[22] F.R.E. De Bisschop, W.J.L. Rigole, A physical model for liquid capillary bridges between adsorptive solid spheres: the nodoid of plateau, J. Colloid Interface Sci. 88 (1982) 117-128.

[23] J.N. Israelachvili, Intermolecular and Surface Forces, second ed., Academic Press, London, 1992.

[24] G. Gupta, P.S. Shah, X. Zhang, A.E. Saunders, B.A. Korgel, K.P. Johnston, Enhanced infusion of gold nanocrystals into mesoporous silica with supercritical carbon dioxide, Chem. Mater. 17 (2005) 6728-6738.

[25] J.E. Lewis, R. Biswas, A.G. Robinson, M. Maroncelli, Local density augmentation in supercritical solvents: electronic shifts of anthracene derivatives, J. Phys. Chem. B 105 (2001) 3306-3318.

[26] G.J. Besserer, D.B. Robinson, Refractive indices of ethane, carbon dioxide, and isobutane, J. Chem. Eng. Data 18 (1973) 137-140.

[27] J. Chen, A. Anandarajah, van der Waals attraction between spherical particles, J. Colloid Interface Sci. 180 (1996) 519-523.

[28] R.A. Bowling, A Theoretical Review of Particle Adhesion, Plenum Press, New York, 1989.

[29] S. Bhattacharya, K.L. Mittal, Mechanics of removing glass particulates from a solid surface, Surf. Technol. 7 (1978) 413-425.

[30] T. Jacobs, Challenge in porous Low-k cleaning, in: Wafer Clean and Surface Prep Workshop 2002, International Sematech, Austin, 2002.

[31] N. Cheng, Solvent Handbook, Chemical Industry Press, Beijing, 2002.

[32] J. Visser, Surface and Colloid Science, John Wiley and Sons, New York, 1976.

[33] C.Y. Day, C.J. Chang, C.Y. Chen, Phase equilibrium of ethanol $+\mathrm{CO}_{2}$ and acetone $+\mathrm{CO}_{2}$ at elevated pressures, J. Chem. Eng. Data 41 (1996) 839-843.

[34] R. Yaginuma, T. Nakajima, H. Tanaka, M. Kato, Densities of carbon dioxide + 2propanol at $313.15 \mathrm{~K}$ and pressures to $9.8 \mathrm{MPa}$, J. Chem. Eng. Data 42 (1997) 814-816.

[35] J. Vorholz, V.I. Harismiadis, B. Rumpf, A.Z. Panagiotopoulos, G. Maurer Vapor + liquid equilibrium of water, carbon dioxide, and the binary system, water + carbon dioxide, from molecular simulation, Fluid Phase Equilib. 170 (2000) 203-234. 
[36] D. Dittmar, S.B. Oei, R. Eggers, Interfacial tension and density of ethanol in contact with carbon dioxide, Chem. Eng. Technol. 25 (2002) 2327.

[37] B.S. Chun, G.T. Wilkinson, Interfacial tension in high-pressure carbon dioxide mixtures, Ind. Eng. Chem. Res. 34 (1995) 4371-4377.

[38] S.R.P. da Rocha, K.L. Harrison, K.P. Johnston, Effect of surfactants on the interfacial tension and emulsion formation between water and carbon dioxide, Langmuir 15 (1999) 419-428.
[39] Y.C. Liao, H.J. Subramani, E.I. Franses, O.A. Basaran, Effects of soluble surfactants on the deformation and breakup of stretching liquid bridges, Langmuir 20 (2004) 9926-9930.

[40] J.L. Dickson, K.P. Johnston, Interfacial properties of fluorocarbon and hydrocarbon phosphate surfactants at the water- $\mathrm{CO}_{2}$ interface, Ind. Eng. Chem. Res. 44 (2005) 1370-1380.

[41] R.C. Weast, CRC Handbook of Chemistry and Physics, 67th ed., CRC Press, Florida, 1986. 